\title{
MR Contrast Agent Safety in the Age of Nephrogenic Systemic Fibrosis: Update 2014
}

\author{
Johannes Budjan • Stefan O. Schoenberg • \\ John N. Morelli $\cdot$ Stefan Haneder
}

Published online: 20 July 2014

(c) Springer Science+Business Media New York 2014

\begin{abstract}
Nephrogenic systemic fibrosis (NFS) is a potentially fatal disease that is linked to the administration of gadolinium based contrast agents (GBCA). As the associated risk factors have been identified and countermeasures taken, NSF has become a rarity. However, there is ongoing research to better understand its pathogenesis and studies on the safety of different GBCA in the context of NSF are carried on. This article aims to provide a brief review of the topic of NSF and to summarize recent developments and literature publications within the last year.
\end{abstract}

Keywords Nephrogenic systemic fibrosis - Contrast media $\cdot$ Gadolinium based contrast agents $\cdot$ Magnetic resonance imaging

\section{Overview}

The first description of nephrogenic systemic fibrosis (NSF) was in 2000, when Cowper et al. [1] reported a new fibrosing dermopathy in patients receiving renal dialysis. These patients presented with skin thickening, hardening,

This article is part of Topical Collection on MRI Safety.

J. Budjan $(\bowtie) \cdot$ S. O. Schoenberg $\cdot$ S. Haneder Institute of Clinical Radiology and Nuclear Medicine, University Medical Center Mannheim, Medical Faculty Mannheim, Heidelberg University, Theodor- Kutzer-Ufer 1-3, 68167 Mannheim, Germany

e-mail: Johannes.Budjan@umm.de

J. N. Morelli

The Russell H. Morgan Department of Radiology and Radiological Science, The Johns Hopkins Hospital, Baltimore, MD, USA and hyperpigmentation typically involving the distal extremities $[1,2]$. Initially referred to as "nephrogenic fibrosing dermopathy," the term was changed to "NSF," as involvement of other organ systems was seen in a subgroup of patients. Since NSF can affect the lungs, heart, and kidneys, it can also occasionally be fatal. In 2006, a possible association between NSF and the administration of gadolinium based contrast agents (GBCA) was postulated for the first time [3]. Extensive work up of the reported NSF cases found it to be limited to patients suffering from advanced renal insufficiency (defined as a glomerular filtration rate $(\mathrm{GFR})<30 \mathrm{~mL} / \mathrm{min} / 1.73 \mathrm{~m} \mathrm{[2])}$ and determined that the risk of developing NSF was linked to the administration of certain GBCAs. Usually the symptoms related to NSF begin within 2-10 weeks after GBCA administration [4-6]. However, there are case-reports of delayed onset NSF up to 8 years later [7,8].

\section{Gadolinium Based Contrast Agents and the Pathophysiology of NSF}

Gadolinium based contrast agents are considered very safe in general with severe adverse effects only rarely observed in large prospective post-marketing surveillance studies [9]. While all GCBA use the paramagnetic effect of the rare earth metal gadolinium to influence image contrast in magnetic resonance imaging (MRI), the GBCAs feature different mechanisms of binding to the gadolinium ions. Since gadolinium is toxic to the human body as a free ion, it is kept in different chelate complexes to prevent a release of free gadolinium. Based on their structure, GBCAs can be classified as linear or macrocyclic. Additionally, GBCAs can be divided into ionic or non-ionic types based on their charge. A correlation between the stability of the 
different compounds, their structure, and charge can be found in vivo: macrocyclic chelates are more stable than linear chelates, and ionic compounds tend to be more stable than non-ionic chelates [10].

Other positively charged ions in the blood plasma such as calcium can competitively bind to the gadolinium-binding site in the chelate compounds. Thus, gadolinium ions can be slowly exchanged with other plasma ions over time in a transmetalation process. The end result is gadolinium ions being released into the human body. Even though the pathophysiology of NSF is not fully understood, studies suggest that free gadolinium ions rather than the GBCA chelate led to the development of NSF [11]. The greater number of unconfounded NSF cases (i.e., cases where only one GBCA has been administered) with linear rather than macrocyclic GBCAs support this theory. However, the structure of the chelate is not the only factor in play: currently, no cases of NSF have been reported for the linear chelate gadobenate dimeglumine (Multihance ${ }^{\circledR}$ ), whereas a large number of cases have been reported with gadopentetate dimeglumine $\left(\right.$ Magnevist $^{\circledR}$ ), a linear chelate demonstrating almost identical thermodynamic stability [10]. This may relate to the $5 \%$ of hepatobiliary excretion that occurs with gadobenate dimeglumine serving a protective effect in renal failure patients. Likewise the rate of NSF with gadobutrol (Gadovist $^{\circledR}$ or Gadavist ${ }^{\circledR}$ ), a macrocyclic compound, is greater than that of gadoterate meglumine (Dotarem ${ }^{\circledR}$ ) and gadoteridol (ProHance $^{\circledR}$ ). Thus, the pathophysiology of NSF appears to be multifactoral. While European guidelines are based predominantly on the model of structural stratification and thermodynamic stability, recent studies suggest a more complex, multifactorial process of inflammation involving cells with osteogenic potential [12] that is triggered by free gadolinium ions.

\section{Risk Factors and Epidemiology of NSF}

Besides gadoxetate disodium and gadobenate dimeglumine, which are partially metabolized by hepatocytes, all GBCA are eliminated almost exclusively by the kidneys. In patients with impaired renal function, especially in those with a GFR $<15 \mathrm{~mL} / \mathrm{min} / 1.73 \mathrm{~m}$ [2], GBCAs have a significantly prolonged plasma time. Thus, GBCAs remain within plasma longer, enabling more time for gadolinium ions to be released from the chelate in patients with renal failure. Additionally, the stability of GBCA is further decreased in low $\mathrm{pH}$ environments [3, 13]. Since renal failure patients typically have a lower blood $\mathrm{pH}$ compared to healthy patients, this may further contribute to the increased NSF risk in these patients [3, 13]. Additionally, low levels of blood phosphorus seem to correlate with the development of NSF [14], although the implication of this possible surrogate marker is not yet understood. In distinction, previous studies had found phosphorus to promote the release of gadolinium from the chelate [10].

In addition to the aforementioned risk factors, higher one-time and cumulative doses of GBCA are considered additional risk factors for the development of NSF. A greater inflammatory burden also increases NSF risk [15]. Liver functional impairment has recently been shown to not be a risk factor in the development of NSF [16, 17••].

Many patients who developed NSF after administration of GBCAs were exposed to different GBCA compounds, thus making it difficult to estimate the effects of an individual GBCA. In patients who received only one GBCA (so-called unconfounded cases), the calculation of the GBCA's individual NSF risk is feasible. Up to now (as of $5 / 2014$ ), the number of unconfounded NSF cases that have been histologically confirmed is fewer than 1,000 [18]. The resulting calculated risk for NSF, accounting for the number of GBCA administrations worldwide, is thus on the order of 0.1-10 NSF cases per one million GBCA administrations in the general population depending on the GBCA. As can be expected from their chemical and physical stability, linear GBCAs are responsible for the majority of these cases.

In a recently published study on the nationwide prevalence of NSF in Denmark, a prevalence of 12 cases per one million inhabitants was found [19]. So far, this represents the highest reported prevalence worldwide, which according to the authors of the study could be explained by the high awareness of NSF in Denmark together with the initiation of this nationwide investigation. An additional study by Nardone et al. assessed the incidence of NSF in pediatric patients, finding only 16 confirmed NSF cases in patients younger than 19 years [20]. As for adults, impaired renal function appears to be the main risk factor in pediatric patients; although, the assessment is further complicated by the fact that the GBCAs approved for pediatric imaging may differ, depending on governmental regulations, from those approved in adults.

\section{Guidelines}

Reacting to the proposed link between NSF and GBCAs, governmental authorities (such as the Food and Drug Association, FDA, and European Medicines Agency, EMEA) and societies (such as the European Society of Urogenital Radiology, ESUR, and the American College of Radiology, ACR) have published regulations and guidelines on this topic. While the major points made in these publications are similar, some differences can be found, especially regarding the use of GBCA in infants and children. As a common theme in these publications, GBCAs are divided into three groups according to their NSF risk. 
As NSF and its associated risk factors have become better understood, minor changes to the respective guidelines have been introduced over the years. Recently, impaired liver function was removed as an individual risk factor from the ESUR guidelines. Additionally, the use of high-risk GBCA in neonates is now contraindicated according to the most recent ESUR guideline [21•].

\section{Overview Over the Available GBCA}

High Risk: Gadodiamide, Gadoversetamide, and Gadopentetate Dimeglumine

As linear chelates, these three GBCAs pose the highest risk of NSF in patients with impaired renal function. Thus, they are contraindicated in patients with a GFR $<30 \mathrm{ml} / \mathrm{min}$ as stated in the ESUR guidelines [21•].

With a calculated risk of 9 NSF cases per one million administrations, gadodiamide poses the highest NSF risk of all GBCA in patients with impaired renal function. Focusing exclusively upon patients with impaired renal function, the incidence of NSF has been estimated at $3-18 \%$ [21•]. If used in a population with unimpaired renal function, however, gadodiamide is considered safe. Thus, it is essential to assess a patient's renal function prior to the administration of gadodiamide.

Gadopentetate dimeglumine is worldwide the most commonly used GBCA and with approximately 115 million examinations performed [22], the GBCA that has been administered the most overall. The reported incidence of NSF in patients with impaired renal function is estimated between 0.1 and $1 \%$ [21 $]$. Despite its high NSF risk, gadopentetate is considered safe with respect to other adverse reactions. As with gadodiamide, the assessment of individual patient risks (i.e., impaired renal function, previous GBCA application) is mandatory prior to the administration of gadopentetate dimeglumine.

While the NSF incidence of gadoversetamide is estimated less than 1 case per one million administrations, gadoversetamide is considered a high-risk GBCA due to its non-ionic linear structure.

\section{Intermediate Risk: Gadoxetate Disodium, Gadofosveset Trisodium, and Gadobenate Dimeglumine}

Gadoxetate disodium is a GBCA that is metabolized equally by the liver and the kidneys. In the liver, it is taken up exclusively by functioning hepatocytes, and is thus useful in the differentiation and characterization of liver lesions [23, 24]. Despite being a linear chelate, it has proven to be highly stable in animal experiments [25]. Further, its alternative mechanism of hepatobiliary excretion might play an important role in patients with impaired renal function, compensating for the reduced renal excretion. So far, no unconfounded cases of NSF after administration of gadoxetate disodium have been reported. Even though a possible risk for patients with both impaired liver and renal function can be hypothesized, there is no data to support this thesis. Likewise, a recent study found no increased risk of NSF in patients suffering from impaired liver function in combination with other potential NSF risk factors $[17 \bullet \bullet]$.

Gadofosveset features a high affinity to albumin. Its renal excretion is thus slower in comparison to other GCBAs, which leads to gadofosveset's relatively long blood half-life of approximately $18 \mathrm{~h}$. Gadofosveset is a linear ionic chelate that is excreted predominantly via the kidneys with minimal hepatobiliary elimination (5\%). By taking advantage of its long blood pool time, Gadofosveset can be used in relatively low concentrations for certain applications, especially in MR angiography [26, 27]. In such settings, examinations can be performed using only half of the gadolinium dose as compared to conventional GBCA. As Gadofosveset was recently re-introduced to the market, questions about its safety have come back to focus. In this context, Alhadad et al. recently published retrospective data on 62 patients who received gadofosveset [28•]. Including a mixed population, no severe adverse reaction, and in particular no cases of NSF were encountered over the observation period of up to 4 years. This supports the previous data, and so far, there are no unconfounded NSF cases in patients who received gadofosveset.

Gadobenate dimeglumine is an ionic linear chelate with low levels of albumin binding and $5 \%$ hepatobiliary elimination that can be used for liver imaging. So far, no unconfounded cases of NSF after administration of gadobenate dimeglumine have been reported.

Low risk: Gadobutrol, Gadoterate Meglumine, and Gadoteridol

The GBCAs with minimal or low risk of NSF are based on cyclic chelates. With their superior stability in comparison to the linear chelates, the administration of these GBCAs poses a relatively low risk to patients with impaired renal function. However, macrocyclic chelates should always be used at lowest possible dosages. A 1 week interval between two consecutive examinations is considered sufficient $[21 \bullet]$.

There is one case demonstrating a possible association between the administration of gadoterate meglumine and NSF [29]. Otherwise, there are no definite unconfounded cases of NSF with the compound so far $[21 \bullet, 29]$. In a recently published prospective study [30], gadoterate 
meglumine was administered in high-risk patients and the rate of NSF cases compared to patients receiving noncontrast MRI scans. In a follow-up time of 3 months, no cases of NSF were recorded in either patient group. Another prospective trial recently assessed the incidence of NSF in patients undergoing long-term dialysis. That study included 268 patients given predominantly macrocyclic GBCAs, usually gadoterate meglumine, and did not report any cases of NSF in these high-risk patients [31•].

Gadoteridol and gadobutrol, the other available cyclic compounds are likewise considered chemically and physically stable and suitable for use even in patients with impaired renal function. When utilized in single doses, they seem to pose no NSF risk to these patients, as was specifically shown for gadoteridol [32].

Overall, the high-risk, linear chelates have been used in much larger numbers relative to the macrocyclic compounds. This greater number of administrations of the highrisk GBCAs has to be taken into consideration. Over the last several years, the total number of administrations for both groups has converged, as the use of low-risk GBCA continues to increase. In the end, a risk classification according to the NSF incidence for the individual GBCA might be considered rather than an assignment based on the chemical structure. This would lead to some changes, as, for example, the linear compound gadobenate dimeglumine would be classified as a low-risk GBCA, as so far, no NSF cases have been found. Table 1 gives an overview over the currently available GBCAs.

\section{Prevention and Treatment of NSF}

Currently, there is no definitive treatment for NSF. Therefore, prevention through a priori risk assessment and the risk stratification of the patients is the best way to approach the disease. The radiologist plays an essential role in managing at risk patients and in reminding clinical colleagues of such risks. By assessing the main risk factors for NSF in patients undergoing contrast-enhanced MRI, i.e., impaired renal function, and excluding those patients from administration of high-risk GBCA, NSF can be effectively prevented.

Additionally, the use of macrocyclic compounds that are considered low-risk GBCA further decreases the risk of NSF, especially when administered in low doses. According to the current guidelines, patients in whom contrastenhanced MRI is indicated should not be excluded due to impaired renal function and can be safely scanned utilizing a low-risk GBCA at the appropriate dose.

Although there is no definitive treatment for NSF, improving renal function seems to be one key. This is supported by data on NSF patients who recovered from acute renal failure or underwent kidney transplantation [33] and subsequently demonstrated a clinical improvement. Therapies resulting in the elimination of free gadolinium ions is another potential option, as a decrease of dermal gadolinium deposition has been correlated with an improvement of clinical symptoms [34]. Different therapeutic approaches such as extracorporeal photopheresis or apheresis seem to be feasible

Table 1 Overview over the different commercially available GBCAs. Adapted from [6, 21, 29]

\begin{tabular}{|c|c|c|c|c|c|c|c|}
\hline $\begin{array}{l}\text { NSF risk } \\
\text { according to } \\
\text { EMA/ESUR }\end{array}$ & Name & Brand name & Manufacturer & $\begin{array}{l}\text { Chelate/ } \\
\text { Charge }\end{array}$ & $\begin{array}{l}\text { FDA- } \\
\text { Approval }\end{array}$ & $\begin{array}{l}\text { Global } \\
\text { administrations }\end{array}$ & $\begin{array}{l}\text { NSF cases/ } \\
1 \text { million } \\
\text { applications }\end{array}$ \\
\hline \multirow[t]{3}{*}{ High } & Gadodiamide & Omniscan ${ }^{\circledR}$ & Rodiag & $\begin{array}{l}\text { Linear/ } \\
\text { non-ionic }\end{array}$ & 1993 & 47 million & 9.3 \\
\hline & Gadoversetamide & Optimark ${ }^{\circledR}$ & $\begin{array}{l}\text { Mallinckrodt } \\
\text { Pharmaceuticals }\end{array}$ & $\begin{array}{l}\text { Linear/ } \\
\text { non-ionic }\end{array}$ & 1999 & $>9$ million & 0.8 \\
\hline & $\begin{array}{l}\text { Gadopentetate } \\
\text { dimeglumine }\end{array}$ & Magnevist $^{\circledR}$ & Bayer HealthCare & Linear/ionic & 1988 & 115 million & 1.2 \\
\hline \multirow[t]{3}{*}{ Intermediate } & $\begin{array}{c}\text { Gadoxetate } \\
\text { disodium }\end{array}$ & $\begin{array}{c}\text { Primovist }^{\circledR} \text {, } \\
\text { Eovist }\end{array}{ }^{\circledR}$, & Bayer HealthCare & Linear/ionic & 2008 & $\mathrm{n} / \mathrm{a}$ & \\
\hline & $\begin{array}{l}\text { Gadofosveset } \\
\text { trisodium }\end{array}$ & $\begin{array}{c}\text { Vasovist }^{\circledR}, \\
\text { Ablavar }^{\circledR}\end{array}$ & $\begin{array}{l}\text { Bayer HealthCare, EPIX } \\
\text { Pharmaceuticals }\end{array}$ & Linear/ionic & 2008 & $\mathrm{n} / \mathrm{a}$ & \\
\hline & $\begin{array}{l}\text { Gadobenate } \\
\text { dimeglumine }\end{array}$ & MultiHance $^{\circledR}$ & Bracco imaging & Linear/ionic & 2004 & $>11$ million & $<0.1$ \\
\hline \multirow[t]{3}{*}{ Low } & Gadobutrol & $\begin{array}{l}\text { Gadovist }^{\circledR}, \\
\text { Gadavist }^{\circledR}\end{array}$ & Bayer HealthCare & $\begin{array}{c}\text { Macrocyclic/ } \\
\text { non-ionic }\end{array}$ & 2011 & $>6$ million & 0.7 \\
\hline & $\begin{array}{l}\text { Gadoterate } \\
\text { meglumine }\end{array}$ & $\begin{array}{l}\text { Dotarem }^{\circledR}, \\
\text { Magnescope }^{\circledR}\end{array}$ & Guerbet & $\begin{array}{l}\text { Macrocyclic/ } \\
\text { ionic }\end{array}$ & 2013 & $>21$ million & $<0.1$ \\
\hline & Gadoteridol & ProHance $^{\circledR}$ & Bracco imaging & $\begin{array}{l}\text { Macrocyclic/ } \\
\text { non-ionic }\end{array}$ & 1992 & $>14$ million & $<0.1$ \\
\hline
\end{tabular}


for the treatment of NSF. Recently, Poisson et al. published a case report of 2 NSF patients whose symptoms improved following therapeutic plasma exchange [35]. Additionally, immunosuppressants and immune modulation are considered therapeutic options in patients suffering from NSF.

Whether or not dialyzing patients who received GBCAs helps to minimize the risk of NSF which has not been definitively proven. As previously shown by several studies, gadopentetate dimeglumine, gadodiamide, and gadobutrol can be removed from the plasma by hemodialysis [36-38]. In a recent study, Gheuens et al. examined the dialyzability of gadoteric acid. By applying a dialysis regimen, they found gadoteric acid to be effectively removed from plasma [39]. Thus, dialysis following administration of gadolinium chelates may prove a useful preventative or therapeutic measure.

\section{Conclusions}

NSF can be effectively prevented by identifying associated risk factors and avoiding certain GBCAs in patients with such risk factors. Although the number of NSF cases is small and will likely further decrease, there remains a need for further research to understand its pathogenesis. In doing so, both NSF as well as newly realized complications, such as long-term alterations to certain brain areas reported by Kanada et al. [40], can be better understood. Technical improvements and the increased clinical use of alternative or functional MR imaging techniques such as DWI, BOLD, etc., might decrease or render unnecessary the clinical use of GBCAs in the future.

\section{Compliance with Ethics Guidelines}

Conflict of Interest Dr. Johannes Budjan, Prof. Dr. Stefan O. Schoenberg, Dr. John N. Morelli, and Dr. Stefan Haneder each declare no potential conflicts of interest.

Human and Animal Rights and Informed Consent This article does not contain any studies with human or animal subjects performed by any of the authors.

\section{References}

Papers of particular interest, published recently, have been highlighted as:

- Of importance

•- Of major importance

1. Cowper SE, Robin HS, Steinberg SM, Su LD, Gupta S, LeBoit PE. Scleromyxoedema-like cutaneous diseases in renal-dialysis patients. Lancet. 2000;356(9234):1000-1.
2. Cowper SE, Rabach M, Girardi M. Clinical and histological findings in nephrogenic systemic fibrosis. Eur J Radiol. 2008;66(2): 191-9.

3. Grobner T. Gadolinium-a specific trigger for the development of nephrogenic fibrosing dermopathy and nephrogenic systemic fibrosis? Nephrol Dial Transplant. 2006;21(4):1104-8.

4. Perez-Rodriguez J, Lai S, Ehst BD, Fine DM, Bluemke DA. Nephrogenic systemic fibrosis: incidence, associations, and effect of risk factor assessment-report of 33 cases. Radiology. 2009;250(2):371-7.

5. Broome DR, Girguis MS, Baron PW, Cottrell AC, Kjellin I, Kirk GA. Gadodiamide-associated nephrogenic systemic fibrosis: why radiologists should be concerned. AJR Am J Roentgenol. 2007;188(2):586-92.

6. Daftari Besheli L, Aran S, Shaqdan K, Kay J, Abujudeh H. Current status of nephrogenic systemic fibrosis. Clin radiol. 2014.

7. Do JG, Kim YB, Lee DG, Hwang JH. A case of delayed onset nephrogenic systemic fibrosis after gadolinium based contrast injection. Ann Rehabil Med. 2012;36(6):880-6.

8. Thakral C, Alhariri J, Abraham JL. Long-term retention of gadolinium in tissues from nephrogenic systemic fibrosis patient after multiple gadolinium-enhanced MRI scans: case report and implications. Contrast Media Mol Imaging. 2007;2(4):199205.

9. Maurer M, Heine O, Wolf M, Durmus T, Wagner M, Hamm B. Tolerability and diagnostic value of gadoteric acid in the general population and in patients with risk factors: results in more than 84,000 patients. Eur J Radiol. 2012;81(5):885-90.

10. Frenzel T, Lengsfeld P, Schirmer H, Hutter J, Weinmann HJ. Stability of gadolinium-based magnetic resonance imaging contrast agents in human serum at 37 degrees C. Invest Radiol. 2008;43(12):817-28.

11. Sieber MA, Steger-Hartmann T, Lengsfeld P, Pietsch H. Gadolinium-based contrast agents and NSF: evidence from animal experience. J Magn Reson Imaging. 2009;30(6): $1268-76$

12. Swaminathan S, Bose C, Shah SV, Hall KA, Hiatt KM. Gadolinium contrast agent-induced CD163 + ferroportin + osteogenic cells in nephrogenic systemic fibrosis. Am J Pathol. 2013;183(3): 796-807.

13. Chopra T, Kandukurti K, Shah S, Ahmed R, Panesar M. Understanding nephrogenic systemic fibrosis. Int $\mathrm{J}$ Nephrol. 2012;2012:912189.

14. Bernstein EJ, Isakova T, Sullivan ME, Chibnik LB, Wolf M, Kay J. Nephrogenic systemic fibrosis is associated with hypophosphataemia: a case-control study. Rheumatology 2014.

15. Sadowski EA, Bennett LK, Chan MR, et al. Nephrogenic systemic fibrosis: risk factors and incidence estimation. Radiology. 2007;243(1):148-57.

16. Hanna RF, Finkelstone LA, Chow DS, et al. Nephrogenic systemic fibrosis risk and liver disease. Int J Nephrol. 2014;2014: 679605.

17. $\bullet$ Smorodinsky E, Ansdell DS, Foster ZW, et al. Risk of nephrogenic systemic fibrosis is low in patients with chronic liver disease exposed to gadolinium-based contrast agents. Journal of magnetic resonance imaging : JMRI 2014. The findings of this study suggest that impaired liver function indeed does not lead to an additionally increased risk of NSF. Up to now, this study is the most comprehensive that deals with the topic of impaired liver function, NSF and GBCA.

18. Weller A, Barber JL, Olsen OE. Gadolinium and nephrogenic systemic fibrosis: an update. Pediatr Nephrol. 2013. doi:10.1007/ s00467-013-2636-z.

19. Elmholdt TR, Olesen AB, Jorgensen B, et al. Nephrogenic systemic fibrosis in Denmark-a nationwide investigation. PLoS One. 2013;8(12):e82037. 
20. Nardone B, Saddleton E, Laumann AE, et al. Pediatric nephrogenic systemic fibrosis is rarely reported: a RADAR report. Pediatr Radiol. 2014;44(2):173-80.

21. - Thomsen HS, Morcos SK, Almen T, et al. Nephrogenic systemic fibrosis and gadolinium-based contrast media: updated ESUR Contrast Medium Safety Committee guidelines. European radiology 2013; 23(2): 307-18. The ESUR guideline summarizes the current knowledge on NSF and provides instructions for clinical practice.

22. Matsumura T, Hayakawa M, Shimada F, et al. Safety of gadopentetate dimeglumine after 120 million administrations over 25 years of clinical use. Magn Reson Med Sci. 2013;12(4): 297-304.

23. Purysko AS, Remer EM, Veniero JC. Focal liver lesion detection and characterization with GD-EOB-DTPA. Clin Radiol. 2011;66(7):673-84.

24. Zech CJ, Herrmann KA, Reiser MF, Schoenberg SO. MR imaging in patients with suspected liver metastases: value of liver-specific contrast agent Gd-EOB-DTPA. Magn Reson Med Sci. 2007;6(1):43-52.

25. Sato T, Ito K, Tamada T, et al. Tissue gadolinium deposition in renally impaired rats exposed to different gadolinium-based MRI contrast agents: evaluation with inductively coupled plasma mass spectrometry (ICP-MS). Magn Reson Imaging. 2013;31(8): 1412-7.

26. Rapp JH, Wolff SD, Quinn SF, et al. Aortoiliac occlusive disease in patients with known or suspected peripheral vascular disease: safety and efficacy of gadofosveset-enhanced MR angiographymulticenter comparative phase III study. Radiology. 2005;236(1): 71-8.

27. Steger-Hartmann T, Graham PB, Muller S, Schweinfurth H. Preclinical safety assessment of Vasovist (Gadofosveset trisodium), a new magnetic resonance imaging contrast agent for angiography. Invest Radiol. 2006;41(5):449-59.

28. - Alhadad A, Akesson M, Lehti L, et al. Safety aspects of gadofosveset in clinical practice-analysis of acute and longterm complications. Magnetic resonance imaging 2014. This study on the safety of gadofosveset in patients with impaired renal function is of importance especially since it was lately reintroduced to the market and is now again available for clinical use.

29. Heverhagen JT, Krombach GA, Gizewski E. Application of Extracellular Gadolinium-based MRI Contrast Agents and the Risk of Nephrogenic Systemic Fibrosis. RoFo. 2014;186(7): 661-9.
30. Deray G, Rouviere O, Bacigalupo L, et al. Safety of meglumine gadoterate (Gd-DOTA)-enhanced MRI compared to unenhanced MRI in patients with chronic kidney disease (RESCUE study). Eur Radiol. 2013;23(5):1250-9.

31. Amet S, Launay-Vacher V, Clement O, et al. Incidence of nephrogenic systemic fibrosis in patients undergoing dialysis after contrast-enhanced magnetic resonance imaging with gadolinium-based contrast agents: the Prospective Fibrose Nephrogenique Systemique study. Investigative radiology 2014; 49(2): 109-15. This is one of the few studies that report on the safety of $G B C A$ in patients who received GBCAs and underwent dialysis prospectively. Another strength is its relatively high number of included patients.

32. Reilly RF. Risk for nephrogenic systemic fibrosis with gadoteridol (ProHance) in patients who are on long-term hemodialysis. Clin J Am Soc Nephrol. 2008;3(3):747-51.

33. Cuffy MC, Singh M, Formica R, et al. Renal transplantation for nephrogenic systemic fibrosis: a case report and review of the literature. Nephrol Dial Transplant. 2011;26(3):1099-101.

34. Schad SG, Heitland P, Kuhn-Velten WN, Gross GE, Jonas L. Time-dependent decrement of dermal gadolinium deposits and significant improvement of skin symptoms in a patient with nephrogenic systemic fibrosis after temporary renal failure. J Cutan Pathol. 2013;40(11):935-44.

35. Poisson JL, Low A, Park YA. The treatment of nephrogenic systemic fibrosis with therapeutic plasma exchange. J Clin Apheresis. 2013;28(4):317-20.

36. Saitoh T, Hayasaka K, Tanaka Y, Kuno T, Nagura Y. Dialyzability of gadodiamide in hemodialysis patients. Radiat Med. 2006;24(6):445-51.

37. Ueda J, Furukawa T, Yamamoto T, Sakaguchi K, Araki Y. Permeability of gadolinium-DTPA through two types of hemodialysis membrane. Invest Radiol. 1998;33(10):734-7.

38. Tombach B, Bremer C, Reimer P, et al. Using highly concentrated gadobutrol as an MR contrast agent in patients also requiring hemodialysis: safety and dialysability. AJR Am J Roentgenol. 2002;178(1):105-9.

39. Gheuens E, Daelemans R, Mesens S. Dialysability of Gadoteric Acid in Patients With End-Stage Renal Disease Undergoing Hemodialysis. Invest radiol. 2014;49(8):505-8.

40. Kanda T, Ishii K, Kawaguchi H, Kitajima K, Takenaka D. High signal intensity in the dentate nucleus and globus pallidus on unenhanced T1-weighted MR images: relationship with increasing cumulative dose of a gadolinium-based contrast material. Radiology. 2014;270(3):834-41. 